\section{Space Instrumentation: Imaging Interferometry}

Due to the degrading effects of the Earth's turbulent atmosphere, the spatial resolution achieved by ground-based optical astronomy is limited to the extent of the SEEING disk - the image of a point source (e.g. a single star), taken through the atmosphere. The size of the Seeing disk is independent of telescope diameter, but changes only with wavelength and climatic conditions - about $0.5^{\prime \prime}$ at the best observing sites in good weather. In the absence of atmospheric Seeing, e.g. in space, or with a perfect ADAPTIVE OPTICS system, a telescope has a resolution defined by the DIFFRACTION LIMIT, which scales inversely with telescope diameter: the larger the mirror, the sharper the image. For example, the HuBBle SPACE Telescope with its $2.5 \mathrm{~m}$ aperture has a diffraction limit of $0.05^{\prime \prime}$ at a wavelength of $500 \mathrm{~nm}$, ten times better than the Seeing limit, but still not good enough. Projects such as resolving the immediate surroundings of black holes in external galaxies, or imaging extra-solar planets, require telescope diameters of at least $100 \mathrm{~m}$. To manufacture and launch into space such large apertures is clearly out of reach of present day technology. Yet, images with such seemingly impossible resolution are feasible. They can be obtained with a method called SYNTHESIS IMAGING which can achieve basically unlimited resolution without unreasonably large telescopes. In this article, I discuss the principle of synthesis imaging and the advantages of imaging interferometry from space. I also briefly describe various projects planned for the near future which promise to revolutionize astronomical imaging. While the general discussion of synthesis imaging is valid for the entire electro-magnetic spectrum, the degree of difficulty of its realization varies dramatically with wavelength. Here, I will focus solely on the optical regime, and ignore the rich and successful history of radio interferometry, which is discussed elsewhere in this volume.

\section{The Idea of Synthesis Imaging}

Figure 1a shows a simple star cluster as it would be seen by a (hypothetical) diffraction-free $10 \mathrm{~m}$ telescope in space. Imagine its primary mirror covered by an opaque mask with a set of holes along a line. Obviously, most of the image information would be lost - but not all of it! The image produced through such a mask shows a set of INTERFERENCE fringes - alternating stripes of high and low intensity (Fig. 1b). These fringes contain information about the structure of the object. Different rotation angles of the mask produce additional fringe patterns. All patterns can be recorded and combined later to obtain the complete source image, as demonstrated in Figs. 1c and 1d. In fact, there is no need for the full $10 \mathrm{~m}$ telescope mirror: the fringe patterns can be obtained with just a set (at least two) of small mirrors by combining their beams in a common focus. This is the elegant principle behind synthesis imaging: to mimic a large telescope with a number of smaller ones.

There is, however, one caveat which in the past has prevented the successful realization of synthesis imaging at optical wavelengths. In order to produce the all-important interference fringes, the beams from the individual apertures must be combined coherently. This means that the difference in their respective pathlengths to the common focus must not exceed the COHEREnCE LENGTH of the light. Combining the two signals coherently requires accurate control of the pathlengths between the two telescopes and the beam combination optics. The enormous technological difficulties associated with controlling optical pathlengths to the required levels of a few wavelengths are described elsewhere in this volume.

\section{How does it work?}

The mathematical framework of synthesis imaging is based on the theory of wave diffraction. The basic result is summarized in the VAN Cittert - Zernike theorem: 
the complex degree of coherence is equal to the normalized Fourier transform of the source intensity distribution'.

The degree of coherence is a complex quantity, the observables measured in practice are its amplitude and phase. They can be derived from the interference pattern (the fringe) which appears on the detector when scanning the delay between the two beams.

An imaging interferometer measures the degree of coherence across the $(u, v)$-plane. Because, in essence, this measures the Fourier transform of the source structure, the vector between the two telescopes - the baseline $\vec{B}$ - is usually measured in the spatial frequency domain, i.e. in units of wavelengths, $u=$ $B_{x} / \lambda$ and $v=B_{y} / \lambda$. The plane in which the source wavefront is sampled is therefore called the $(u, v)$-plane. Different baseline vectors are achieved by moving the individual telescopes with respect to the source, thus sampling different $(u, v)$-points. According to the van Cittert - Zernike theorem, this allows the full reconstruction of the source structure via an inverse Fourier transform, provided that the $(u, v)$-coverage is complete. However, the $(u, v)$ coverage of any real interferometer will have gaps, resulting in ambiguity in the source reconstruction. The ring-like residuals in Fig. 1d are one example for such ambiguities, also known as GRATING RINGS. The completeness of the $(u, v)$-coverage, is therefore a critical design parameter for imaging interferometers.

\section{Spatial resolution}

To first order, the resolution of an imaging interferometer is defined by the longest baseline. However, in contrast to the diffraction limit of a filled aperture, the spatial resolution of a syn-

\footnotetext{
1 Strictly speaking, this formulation of the van Cittert - Zernike theorem is correct only for the case of an incoherent light source. Also, both the source size and the baseline length must be much smaller than the distance to the source (the far-field approximation). However, astronomical sources at optical wavelengths always meet all of these conditions.
}

thesis imaging observation is not uniquely defined. By varying the weighting of the data obtained at different $(u, v)$-coordinates, or even removing some baselines completely, one can put more or less emphasis on certain spatial frequencies in the reconstruction, and thus change the effective resolution, even after the data are taken. This fact is regularly exploited at ground-based telescopes in APERTURE MASKING observations of bright sources for which collecting area is not an issue, but the highest possible resolution is required.

\section{Field of View}

The field of view (FOV) of an imaging interferometer is limited by the coherence length of the light, $l=\lambda^{2} / \Delta \lambda$, where $\lambda$ and $\Delta \lambda$ are the wavelength and the bandwidth of the observation. While the path lengths from the two interferometer elements ideally are equal in the center of the field, an angular separation $\Theta$ from the center necessarily produces a path difference $\Theta \cdot D$. As long as this difference is small compared to $l$, the fringe contrast will not be affected. Thus, $\Theta=<\frac{\lambda}{D} \cdot \frac{\lambda}{\Delta \lambda}$, or $\Theta<R_{\text {spatial }} \cdot R_{\text {spectral }}$. The maximum FOV of an interferometer thus can be estimated from its spatial and spectral resolution. In practice, however, the FOV is likely to be limited by the effects of the incomplete $(u, v)$-coverage (see Fig. 1).

\section{Synthesis Imaging in Space}

All that has been said so far about synthesis imaging applies equally to space- and groundbased interferometers. There are, however, important differences between the two environments in a number of aspects:

$(u, v)$-coverage: Most interferometer arrays will consist of relatively few elements because of cost constraints. In order to overcome the intrinsically sparse $(u, v)$-coverage of such systems, many employ movable apertures. In addition, all ground-based arrays 
use the Earth's rotation during the course of the night to sample many different $(u, v)$ points. The achievable $(u, v)$-coverage therefore depends both on the geographical location of the array, and the source position with respect to the Earth's rotation axis.

In contrast, an interferometer in space cannot rely on the Earth's rotation. It therefore must have movable apertures to fill the $(u, v)$ plane. On the other hand, the $(u, v)$-coverage can be chosen freely. This allows a uniform resolution which can be valuable in survey programs which study the statistical properties of large numbers of similar objects.

Aperture size: The size of the individual apertures of a ground-based interferometer is limited to the size of an atmospheric turbulence cell - about $30 \mathrm{~cm}$ at optical wavelengths. In principle, the use of Adaptive Optics can overcome this limit, but the need for a bright reference source (a "guide star") within the isoplanatic patch severely limits the number of suitable targets.

In space, on the other hand, there is no turbulent atmosphere. Therefore, the size of interferometer elements is limited only by our technical and financial ability to manufacture - and bring into space - large mirrors.

Frame time: Another important obstacle for interferometry on the ground imposed by the earth's atmosphere is the need to record the complex visibility within a coherence time of the atmosphere - about $20 \mathrm{~ms}$ at a good site for optical wavelengths. Together with the above limit on aperture size, this severely constrains the target brightness: it has to be bright enough to overcome the noise associated with the detection system, both from the detector and the read-out electronics.

Even with a perfect Adaptive Optics system, the integration time at each $(u, v)$-point is limited because of the Earth's rotation: the measurement has to be completed before the baseline has changed noticably in order to avoid $(u, v)$-smearing.

In space, the available integration time for each $(u, v)$-coordinate is in principle unlimited, so that much fainter objects can be observed. This assumes, however, that structural vibrations or slow drifts are perfectly corrected which might not be the case.

Passbands: Astronomy from the ground is limited to a number of small regions of the electro-magnetic spectrum over which the Earth's atmosphere is transparent. In space, no such limitations exist, and important wavebands such as the ultraviolet or the midinfrared become accessible.

Environment: Clearly, operating an interferometer in space to the extreme precision required for successful fringe tracking, is an extremely challenging task. In the case of single spacecraft with a number of apertures on a connecting truss, the mechanical vibrations of the structure must be minimized, and even for low-noise structures, residual motions must be actively corrected. In principle, however, space is a favorable environment because it is intrinsically much quieter than the geologically active surface of the Earth. Because of this and the limitations of a truss with regard to the achievable baseline length, future synthesis imaging in space is likely to be realized with a suite of free-flying apertures which are positionally controlled with respect to each other and the beam combining optics by reference laser beams.

\section{Ongoing and Planned Projects}

The technology which makes the ambitious methods of active pathlength control and fringe tracking feasible has only recently become available, mostly through the development of ever faster computers which allow real-time control of mechanical instabilities. While the principles of synthesis imaging are well understood, and the technique has been demonstrated on the ground, interferometry in space has yet to be realized. Today, a number of space-based interferometer projects are in the development or planning stages as milestones of NASA's Origins Program. Most of these 
missions will be capable of performing synthesis imaging:

Space Technology 3 (ST3) is intended to demonstrate the feasibility of precision control for free-flying spacecrafts. It will consist of two spacecrafts, one with a collector and the beam combining optics, and one that serves only as a collector. Launch is planned for 2003.

Space Interferometry Mission (SIM) consists of a single spacecraft with a $12 \mathrm{~m}$ truss which contains eight telescopes, each with a diameter of about $30 \mathrm{~cm}$. SIM primary science goal is to perform high-precision astrometry for a number of science programs. Because SIM will allow to rotate the truss structure, and to combine any pair of two out of the eight telescopes, it has great potential for synthesis imaging. SIM will be launched in 2006.

Terrestrial Planet Finder (TPF) in its current design comprises four free-flying $3.5 \mathrm{~m}$ telescopes and a fifth spacecraft with the beamcombining optics. The design allows maximum baselines of about $1 \mathrm{~km}$ for a resolution of less than $0.001^{\prime \prime}$ at wavelengths around $3 \mu \mathrm{m}$. The main scientific goal of TPF is the detection and characterization of Earth-like planets at infrared wavelengths by means of interferometric Nulling of the light from the host star. A tentative launch date for TPF is 2010 .

DARWIN is a mission concept currently proposed for a cornerstone mission of the European Space Agency (ESA) with a launch after 2009. It has similar design concept and scientific goals as TPF.

While the basic technological tools for these ambitious missions are in hand, their successful implementation will occur in steps and require time, effort, and money. On the way to the ultimate goal of taking images of other Earths, major advances in many other aspects of astronomy are almost certain due to the dramatic increase in spatial resolution that only synthesis imaging can provide.

\section{Bibliography}

The complete treatment of wave diffraction and interference is treated in great detail in Born, M. \& Wolf, E. 1980 Principles of Optics (Oxford: Pergamon) 6th Ed.

A more detailed review about the intricacies of interferometry both from the ground and in space can be found in Shao, M. \& Colavita, M. M. 1992, Long baseline optical and infrared stellar interferometry Ann. Rev. of Astronomy \& Astrophysics 30 457-498

TORSTEN BÖKER

Fig. 1 Illustration of the principle of synthesis imaging: a) model source to be imaged. b-d) Image reconstructions (right) for various baseline distributions (left). The ring-like residuals around the central source image are called grating rings. They are a consequence of the regularly spaced gaps in the $(u, v)$-coverage, and can be removed to a large extent by numerical image restoration algorithms. 
This figure "eaa_boeker_fig1.gif" is available in "gif" format from: http://arxiv.org/ps/astro-ph/9912479v1 